\title{
Liver Fibrosis and Progression of Liver Disease in Patients with Hepatitis Delta - Results from a Retrospective Study in Romania
}

\author{
Oana Săndulescu ${ }^{1,2}$, Tamer Samir Al Aker ${ }^{1}$, Anca Streinu-Cercel ${ }^{1,2}$, Adrian Streinu-Cercel $^{1,2}$
}

'Carol Davila University of Medicine and Pharmacy, Bucharest, Romania

${ }^{2}$ National Institute for Infectious Diseases "Prof. Dr. Matei Balș", Bucharest, Romania

\section{ABSTRACT}

Introduction: Due to the relatively low prevalence of hepatitis delta in Europe, Romania is one of the main countries in the region that can provide data on long-term prognosis of patients with this condition.

Methods: We performed a retrospective study to assess liver disease progression and to compare non-invasive methods for assessing liver fibrosis in patients with chronic hepatitis $B$ and delta under current active surveillance at the National Institute for Infectious Diseases "Prof. Dr. Matei Balș", Bucharest, Romania.

Results: The study group included 64 patients with a median age of 54 (IQR: 38, 59) years, and a male-to-female ratio of $0.7: 1$, accounting for 183.6 patient-years of follow-up. We identified a biphasic distribution of liver fibrosis in patients with hepatitis D from our cohort, with two peaks, one at mild to moderate fibrosis and the second at moderate to advanced liver fibrosis. We recorded a significant decrease in thrombocyte count from baseline to the third evaluation (mean decrease $17,000 / \mu \mathrm{L}, p=0.007, Z=-2.7$ ), a pattern not seen when analyzing coagulation and liver function. We recorded no significant changes over time in terms of cytolysis, renal function, fasting plasma glucose, or lipid profile. We identified a moderate correlation between FibroTest and FIB-4 values $(p=0.008, r s=0.40)$, and a weak correlation between FibroTest and APRI values $(p=0.016$, $r s=0.36)$. We also identified a moderate correlation between ActiTest and FIB-4 values $(p=0.006, r s=0.45)$, and for FibroTest and APRI $(p=0.001, r s=0.56)$.

Conclusions: We identified an accelerated decrease in thrombocyte counts in patients with hepatitis delta without advanced liver fibrosis, unparalleled by a decrease in liver function. FIB-4 might offer a more suitable estimate of liver fibrosis than APRI, while both FIB-4 and APRI scores appear to give a fair evaluation of the necroinflammatory activity in patients with hepatitis delta.

Key words: Non-invasive fibrosis assessment, liver fibrosis, hepatitis delta, hepatitis D

\author{
Corresponding author: \\ Anca Streinu-Cercel, MD, PhD \\ Carol Davila University of Medicine \\ and Pharmacy, Bucharest, Romania \\ National Institute for Infectious \\ Diseases "Prof. Dr. Matei Balș" \\ 1 Dr. Calistrat Grozovici street, \\ Bucharest 021105, Romania \\ E-mail: anca_sc@yahoo.com
}




\section{INTRODUCTION}

The worldwide distribution of hepatitis delta is polymorphic, with high prevalences in the Mediterranean region, South America, Central Africa, and the Middle East. (1) Until relatively recently, Romania was considered a high prevalence zone. (2) However, the landscape has changed as a result of consistent newborn vaccination against hepatitis $B$ virus (HBV) in Romania during the past 20 years, (3) which has led to an important decrease in the receptive population for hepatitis delta virus (HDV).

Due to the relatively low prevalence of hepatitis delta in Europe, Romania is one of the main countries in the region that can provide data on long-term prognosis of patients with this condition. For this reason, we have performed a study to assess liver fibrosis and liver disease progression in patients with chronic hepatitis $B$ and delta in Romania.

\section{MATERIAL AND METHOD}

We performed a retrospective study to assess liver disease progression and to compare non-invasive methods for assessing liver fibrosis in patients with chronic hepatitis B and delta under current active surveillance at the National Institute for Infectious Diseases "Prof. Dr. Matei Balș", Bucharest, Romania in 2013.

Subjects were selected from a cohort of 122 patients monitored in the Institute, and were included in this study if they had complete clinical and laboratory data at three different time points, defined as follows: baseline data - the first complete laboratory evaluation after the diagnosis of hepatitis delta (in the timespan 2002 to 2006) - plus two subsequent evaluations in the timespan 2006 to 2013. Laboratory tests included complete blood count and chemistry for hepatic function, renal function, and lipid profile. FIB-4 and APRI scores were calculated based on standardized equations, (4) and compared to FibroTest results as reference. (5)

Statistical analysis was performed with IBM SPSS Statistics for Windows, version 22 (IBM Corp., Armonk, NY, USA). For continuous variables that were nonnormally distributed, we report medians and interquartile ranges (IQR) and the results of Wilcoxon's signed rank test and Spearman's correlations.

\section{RESULTS}

The study group included 64 patients with a median age of 54 (IQR: 38,59 ) years, and a male-to-female ratio of 0.7:1. Our study included a median follow-up of 1.3 years until the second evaluation (IQR 0.5, 2.5 years), and 2.8 (IQR 1.3, 4.9) years until the third evaluation, for a total of 183.6 patient-years of follow-up.

FibroTest values presented two peaks (fig. 1), with most of the patients presenting mild to moderate fibrosis (57.9\% F0 to F2), followed by a second peak (21.1\%) at F3 to F3-F4, while $21.1 \%$ presented cirrhosis, with no significant difference in FibroTest values between genders $(p=0.961, U=151.5)$ - fig. 2. On ActiTest, $30.3 \%$ presented mild to moderate necroinflammatory activity (AO-A1) and $33.3 \%$ high activity

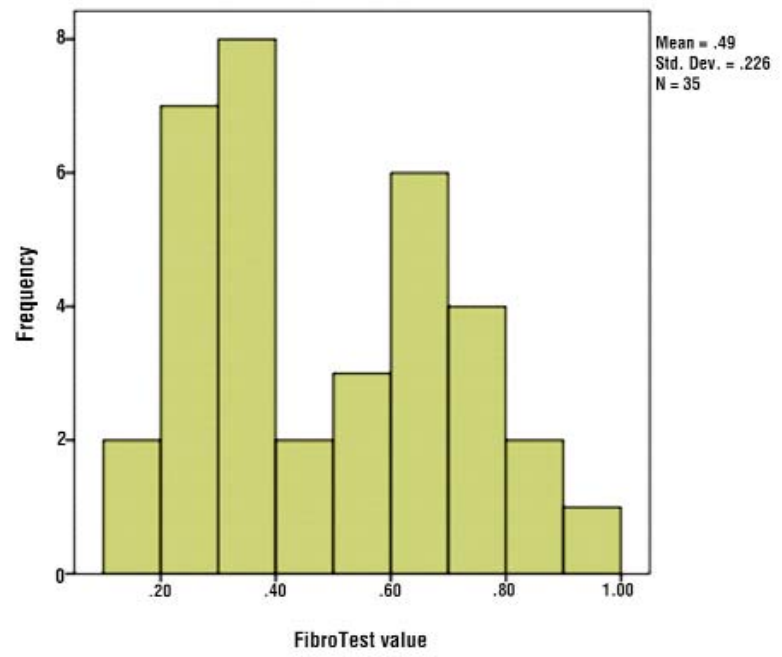

Figure 1 - Distribution of FibroTest values in patients with chronic hepatitis $B$ and delta

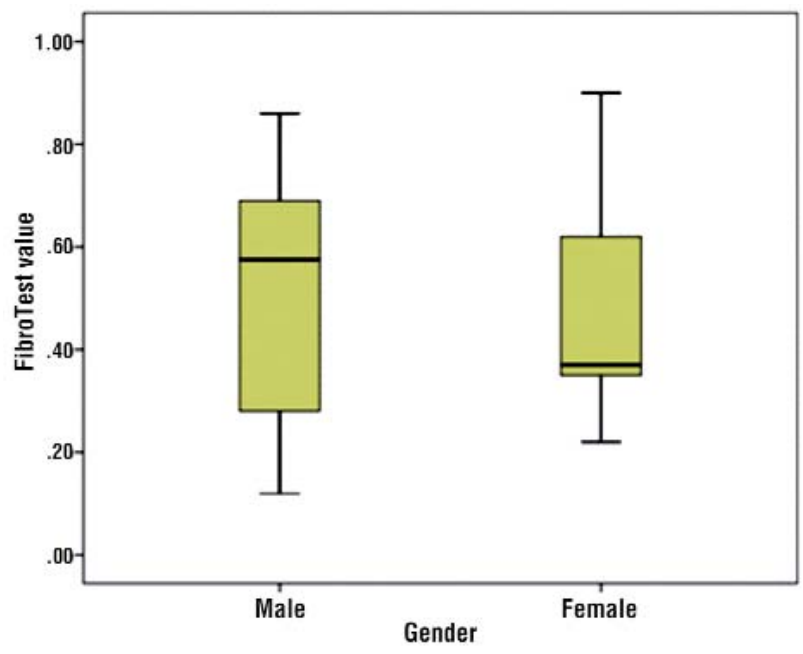

Figure 2 - Boxplot for gender distribution of FibroTest values in patients with chronic hepatitis B and delta 
(A3), with no significant difference in values between genders $(p=0.770, U=112.5)$. On SteatoTest $76.5 \%$ presented mild to moderate steatosis (SO-S2), and $11.8 \%$ borderline high steatosis (S3-S4), and values did not differ significantly between genders $(p=0.277$, $\mathrm{U}=24.5$ ).

We recorded a significant decrease in thrombocyte count from baseline to the third evaluation (mean decrease $17,000 / \mu \mathrm{L}, \mathrm{p}=0.007, \mathrm{Z}=-2.7)$, and a mean decrease of $7,000 / \mu \mathrm{L}$ at the second evaluation, which failed however to reach statistical significance $(p=0.166$, $z=-1.4)$. We recorded no significant variation in terms of liver function translated into coagulation (assessed through prothrombin concentration, which remained steadily at $88.5 \%$ ) or fibrinogen (which remained steadily around $270 \mathrm{mg} / \mathrm{dL}$ ). We recorded a mean decrease in albumin from 3.8 to $1.6 \mathrm{~g} / \mathrm{dL}$, but this difference failed to reach statistical significance $(p=0.180, z=-1.3)$.

We identified no statistically significant variation in terms of cytolysis between the three assessments - a slight increase in ALT was noted, with $11 \mathrm{U} / \mathrm{L}$ by the second evaluation $(p=0.425, z=-0.8)$ and $12.5 \mathrm{U} / \mathrm{L}$ by the third evaluation $(p=0.475, z=-0.7)$. The same was true for fasting plasma glucose (it remained steadily at 90 $\mathrm{mg} / \mathrm{dL}$ ), urea (it remained around $34 \mathrm{mg} / \mathrm{dL}$ ), and creatinine (it remained around $0.8 \mathrm{mg} / \mathrm{dL}$ ). The lipid profile was also not significantly different at subsequent visits, compared with baseline, with median total cholesterol levels at $256 \mathrm{mg} / \mathrm{dL}, \mathrm{HDL}$ cholesterol at $57 \mathrm{mg} / \mathrm{dL}$, and triglycerides at $130 \mathrm{mg} / \mathrm{dL}$. We also did not identify any significant fluctuations in HBV DNA or HDV RNA during the study $(p=0.317, Z=-1.0)$, with median HBV DNA values of $4370 \mathrm{IU} / \mathrm{mL}$ (IQR 1069, 1,291,695 IU/mL) and median HDV RNA values of 30,454 copies/mL (IQR $1242,11,264,296$ copies/mL) at baseline.

We identified a moderate correlation between FibroTest and FIB-4 values ( $\left.p=0.008, r_{s}=0.40\right)$, and a weak correlation between FibroTest and APRI values $\left(p=0.016, r_{s}=0.36\right)$. We also identified a moderate correlation between ActiTest and FIB-4 values $\left(p=0.006, r_{s}=0.45\right)$, and the same was true for FibroTest and APRI $\left(p=0.001, r_{s}=0.56\right)$.

\section{DISCUSSIONS}

Ample data is available in field literature on the evolution of chronic hepatitis $B,(6,7)$ however the longterm progression of liver disease in patients with chronic hepatitis B and delta has been less explored, probably due to the lower prevalence of HDV worldwide. (8)
In our patient population with chronic hepatitis B and delta, we recorded a steady decrease of thrombocyte counts over time, with $7,000 / \mu \mathrm{L}$ in 1.3 years and $17,000 / \mu \mathrm{L}$ in 2.8 years after diagnosing HDV infection. This information is derived from a patient population that already presented some degree of fibrosis at baseline, but notably only $21.1 \%$ of the patients presented cirrhosis. This decrease was not paralleled by an alteration in liver function. HBV and HDV viral loads remained relatively constant over the course of the study, and we recorded no significant changes over time in terms of cytolysis, renal function, fasting plasma glucose, or lipid profile.

In our study, we identified a biphasic distribution of liver fibrosis in patients with hepatitis $D$ from our cohort, with two peaks, one at mild to moderate fibrosis and the second at moderate to advanced liver fibrosis. The proportion of patients who already had cirrhosis at the moment of hepatitis delta diagnosis was lower in our study (21.1\%) compared to the German study $(45.6 \%)$ by Wranke et al. (9) When comparing non-invasive assessments of liver fibrosis, we found that FIB-4 scores correlated moderately with FibroTest values, and that both FIB-4 and APRI scores correlated moderately with ActiTest values.

A limitation of our study is that it is derived from the patient cohort attending one single center in Bucharest Romania, however the Institute is a national referral center and therefore manages a large population of patients. This study's results are interesting as they suggest that patients with chronic hepatitis B who present an accelerated decrease in thrombocyte count in the absence of advanced liver fibrosis may indeed be experiencing superinfection with HDV. However, this hypothesis remains to be confirmed in future studies comparing thrombocyte dynamics in patients with $\mathrm{HBV}+\mathrm{HDV}$ infection and with HBV infection alone. Periodic screening for HDV superinfection in patients with chronic hepatitis B is recommended, and is currently part of routine monitoring in Romania.

\section{CONCLUSIONS}

We identified an accelerated decrease in thrombocyte counts in patients with hepatitis delta without advanced liver fibrosis, unparalleled by a decrease in liver function. FIB-4 might offer a more suitable estimate of liver fibrosis compared with APRI in patients with hepatitis delta, while both FIB-4 and APRI scores appear to give a fair evaluation of the necroinflammatory activity in this category of patients. 


\section{Acknowledgements}

This article is part of the license thesis " $\mathrm{A}$ cohort study in the assessment of liver fibrosis and markers of disease progression in hepatitis B and D coinfection" performed at the Carol Davila University of Medicine and Pharmacy, Bucharest, Romania. Coordinator: Dr. Anca Streinu-Cercel; Supervisor: Dr. Oana Săndulescu.

\section{Authors' contributions}

All authors had equal contributions.

Conflicts of interest: none declared

Source of funding: none

\section{REFERENCES}

1. Wedemeyer H, Manns MP. Epidemiology, pathogenesis and management of hepatitis D: update and challenges ahead. Nat Rev
Gastroenterol Hepatol. 2010 Jan;7(1):31-40. doi: 10.1038/nrgastro.2009.205

2. Abbas Z, Jafri W, Raza S. Scenario in the Asia-Pacific region. World J Gastroenterol. 2010 Feb 7;16(5):554-62.

3. Streinu-Cercel A. Hepatitis B in the spotlight. Germs. 2011 Dec 24; 1(1):5. doi: 10.11599/germs.2012.1001. eCollection 2011.

4. Martinez SM, Crespo G, Navasa M, Forns X. Noninvasive assessment of liver fibrosis. Hepatology. 2011 Jan;53(1):325-35. doi: 10.1002/hep.24013. Epub 2010 Nov 29.

5. Munteanu M. Biomarker panels and regression of fibrosis in chronic viral hepatitis. Germs. 2015 Dec 2;5(4):115. doi: 10.11599/germs. 2015.1078. eCollection 2015.

6. Popescu I, Ionescu M, Brasoveanu V, Hrehort D, Botea F, Dorobantu $B$, et al. Building a liver transplant program in a resource limited country: A 15-year retrospective analysis of 648 patients operated in the Romanian National Program. J. Transl. Med. Res 2015;20(2):5566

7. Borzooy Z, Streinu-Cercel A, Mirshafiey A, Khamseh A, Mahmoudie MK, Navabi SS, et al. IL-17 and IL-22 genetic polymorphisms in HBV vaccine non- and low-responders among healthcare workers. Germs. 2016 Mar 1;6(1):14-20. doi: 10.11599/germs.2016.1084. eCollection 2016.

8. Negro F. Hepatitis D virus coinfection and superinfection. Cold Spring Harb Perspect Med. 2014 Nov 3:4(11):a021550. doi: 10.1101/cshperspect.a021550.

9. Wranke A, Serrano BC, Heidrich B, Kirschner J, Bremer B, Lehmann $P$, et al. Antiviral treatment and liver-related complications in hepatitis delta. Hepatology. 2017 Feb;65(2):414-425. doi: 10.1002/hep.28876. Epub 2016 Nov 30. 